\title{
Nek2C functions as a tumor promoter in human breast tumorigenesis
}

\author{
ZIYU LIU ${ }^{1}$, YAHONG WANG ${ }^{1}$, SHULING WANG ${ }^{1}$, JING ZHANG $^{1}$, FEI ZHANG ${ }^{2}$ and YUN NIU ${ }^{1}$ \\ ${ }^{1}$ Department of Breast Cancer Pathology and Research Laboratory and ${ }^{2}$ Research Center of Basic \\ Medical Sciences, Key Laboratory of Breast Cancer Prevention and Therapy, Ministry of Education, \\ Tianjin Medical University Cancer Institute and Hospital, Tianjin 300060, P.R. China
}

Received March 18, 2012; Accepted June 15, 2012

DOI: $10.3892 /$ ijmm.2012.1069

\begin{abstract}
The serine/threonine kinase Nek2 has been proposed as a requirement for the progression of breast cancer. The aim of this study was to investigate the expression of $\mathrm{Nek} 2 \mathrm{C}$, which is a splice variant of Nek2, and the role it plays in the different stages of breast cancer. We investigated the role of Nek2C in the MCF10 breast cancer cell lines, MCF10A, MCF10AT, MCF10DCIS.com and MCF10CA1a, using RNA interference and plasmid transfection, as well as breast tissue samples of normal breast tissue (NBT), atypical ductal hyperplasia (ADH), ductal carcinoma in situ (DCIS) and invasive ductal carcinoma (IDC). We detected the mRNA Nek2C expression levels in the MCF10 cell lines and in human breast samples. Our results revealed that the mRNA expression of Nek2C was significantly upregulated in the MCF10DCIS.com and MCF10CA1a cell lines as well as in human primary breast cancer tissue (DCIS and IDC). As expected, the Nek2C downregulation, using RNA interference, decreased the survival, invasion and migration of MCF10DCIS.com and MCF10CA1a cells. Consistent with these results, the Nek2C upregulation in MCF10A and MCF10AT cells using plasmid transfection increased the survival ability of these cells. Our results also revealed a correlation between Nek2C mRNA expression levels and tumor grade. Taken together, our findings suggest that Nek2C plays a signicficant role in breast cancer development and that Nek2C inhibition may be a useful therapeutic approach to targeting human breast tumors.
\end{abstract}

\section{Introduction}

Breast cancer is the most common cancer in women and one of the leading causes of morbidity in women between

Correspondence to: Professor Yun Niu, Department of Breast Cancer Pathology and Research Laboratory, Key Laboratory of Breast Cancer Prevention and Therapy, Ministry of Education, Tianjin Medical University Cancer Institute and Hospital, West Huanhu Road, Ti Yuan Bei, Hexi District, Tianjin 300060, P.R. China

E-mail: yunniu2000@126.com

Key words: Nek2C, breast cancer, MCF10 cell lines the ages of 40 and 44 . This malignancy represents a heterogeneous group of tumors with varied morphology, behavior and response to therapy. Nek2 is a serine/threonine kinase of the NIMA-related kinase family that localizes to the centrosomes (1-4), the microtubule-organizing centers of a cell. It is involved in cell division and mitotic regulation by centrosome splitting $(1,2,4)$. When compared to normal breast tissue (NBT), breast carcinoma cells have a much higher frequency of centrosome defects, including amplification of centrosome number, increased volume, and supernumerary centrioles $(5,6)$. According to Liu et al, the elevation of Nek2 contributes to chromosome instability and promotes aneuploidy by disrupting the control of the mitotic checkpoint (7). Nek2 is upregulated in a number of human cancer cell lines, including breast, cervical and prostate carcinomas, and its downregulation inhibits cell proliferation (8-10). Nek2 has 3 splice variants in humans, namely, Nek2A, Nek2B and Nek2C. Nek2A is 445 amino acids in length $(48 \mathrm{kDa})$ and is considered as a full-length protein. Nek2B is 384 amino acids long ( $44 \mathrm{kDa}$ ) and arises through the use of an alternative polyadenylation site within the terminal intron. These characteristics indicate that Nek2A and Nek2B are identical up to residue 370 but differ in their extreme C-termini. Nek2C arises from an alternative splicing event, excising 8 amino acids (371-378) from the C-terminal domain of the full-length protein $(11,12)$. Similar to many protein kinases with roles in mitosis, Nek2C is overexpressed in breast carcinoma in a tumor-specific manner. However, the role of this protein in breast carcinomas has not been fully elucidated.

A comprehensive and consistent depiction of the genetic changes that underlie breast cancer initiation, development and progression has not yet been provided. The MCF10 model includes a series of cell lines, such as the normal immortalized breast cell line (MCF10A), atypical ductal hyperplasia (ADH; MCF10AT), ductal carcinomain situ(DCIS; MCF10DCIS.com) and invasive carcinoma (MCF10CA1a) cell lines. These 4 cell lines resemble the initiation, development and progression steps of breast carcinoma, respectively. Their cytogenetic and molecular variation may help to reveal genetic changes which are relevant to breast cancer (13). MCF10A (a non-transformed, near diploid, spontaneously immortalized human mammary epithelial cell line) has been used as a normal immortalized breast epithelium control for studies of human breast cell lines (14). 
The above studies reported the function of Nek2A and Nek2B in tumor cell lines, however, few studies have focused on the role of Nek2C in breast cancer MCF10 cell lines. In this study, we detected Nek2C mRNA levels not only in MCF10 cell lines (MCF10A, MCF10AT, MCF10DCIS.com and MCF10CA1a), but also in breast tissue, including NBT, ADH, DCIS and invasive ductal carcinoma (IDC) tissues. This study provides information regarding Nek2C mRNA expression in breast tissue and suggests a correlation between Nek2C mRNA expression and the development and progression of breast carcinoma. The results from our study provide evidence that $\mathrm{Nek} 2 \mathrm{C}$ is a novel potential biomarker for the diagnosis and treatment of human breast cancer.

\section{Materials and methods}

Cell lines and cultures. The MCF10A (normal immortalized breast epithelial cell line), MCF10AT (ADH) and MCF10CA1a (malignant breast epithelial) cell lines were purchased from the Barbara Ann Karmanos Cancer Institute of Wayne State University, Detroit, MI, USA. The MCF10DCIS.com cell line (DCIS) was purchased from an Asterand Business Development Representative. The breast cancer cell lines, MCF10DCIS.com and MCF10CA1a, were grown in DMEM/ F12 (1:1) with 5\% horse serum, $0.029 \mathrm{M}$ sodium bicarbonate, $10 \mathrm{mM}$ HEPES, penicillin and streptomycin. The culture media for the MCF10A and MCF10AT series were similar but also included insulin $(10 \mu \mathrm{g} / \mathrm{ml})$, EGF $(20 \mathrm{ng} / \mathrm{ml})$, hydrocortisone $(0.5 \mu \mathrm{g} / \mathrm{ml})$, and cholera toxin $(100 \mathrm{ng} / \mathrm{ml})$. All cell lines were cultured as recommended by the suppliers, and all cell assays were conducted in serum-containing growth medium unless otherwise specified. All cell lines were passaged for $<6$ months in this study.

siRNA interference. The siRNAs targeting Nek2C, obtained from Invitrogen (Beijing, China), are referred to as Nek2C siRNA-1, siRNA-2 and siRNA-3 as follows: Nek2C siRNA-1 sense, UUUGUAAUUACACUAGCCAGAUCCC and antisense, GGGAUCUGGCUAGUGUAAUUACAAA; Nek2C siRNA-2 sense, UUAAUAUUCUAGCUAGCCCAAAGUC and antisense, GACUUUGGGCUAGCUAGAAUAUUAA; Nek2C siRNA-3 sense, CAUUAAUGCACAUAACUCAU ACAGC and antisense, GCUGUAUGAGUUAUGUGCAUU AAUG. Control siRNAs containing non-specific sequences without homologs in the human genome were also provided by Invitrogen (Cat no. 12935-300). The transfection procedure was achieved using Lipofectamine ${ }^{\mathrm{TM}}$ RNAiMAX reagent(Invitrogen, Carlsbad, CA, USA) according to the manufacturer's instructions. Seventy-two hours after transfection using the Lipofectamine ${ }^{\mathrm{TM}}$ PLUS transfection reagent (Invitrogen), MCF10DCIS.com and MCF10CA1 a cells were harvested and used for further experiments.

Plasmid transfection. The Nek2C expression plasmid (pcDNAmycNek2C) was generated by cloning its coding region into the pcDNA3.0-myc vector (Invitrogen, Beijing, China) and the pcDNA3.0-myc vector was used as the control. For DNA transfections, MCF10A and MCF10AT cells were plated at 300,000 cells/well in 6-well plates 2 days prior to transfection and cultured to $70 \%$ confluence the following day in $5 \%$ horse serum. The following day, cells were transfected with pcDNA3.1-Nek2C or with the empty vector as the control according to the manufacturer's instructions using Lipofectamine 2000 (Invitrogen). Seventy-two hours after transfection, the cells were harvested and used for further experiments.

Semiquantitative reverse transcription-PCR (RT-PCR). Total-RNA was isolated from cultured cells using TRI reagent (Invitrogen) according to the manufacturer's instructions. RNA was treated with DNase I RNase-free (Roche) and purified over an RNeasy column. The first-strand complementary DNA was synthesized with oligo(dT) primer by using the Reverse Transcription System (Promega Biotech, Beijing, China). PCR amplification of Nek2C-specific fragments of $129 \mathrm{bp}$ was performed in a $20 \mu \mathrm{l}$ reaction using TaqDNA polymerase (Invitrogen), with $1 \mu \mathrm{l}$ of the first-strand cDNA synthesis mixture as the template, and the following primers: 1077F, 5'-GGAACGGAAGTTCCTGTC-3'; and 1229R, 5'-CACTTGGACTTAGATGTGA-3'. The conditions for PCR reactions were as follows: $95^{\circ} \mathrm{C}$ for $5 \mathrm{~min}, 95^{\circ} \mathrm{C}$ for $15 \mathrm{sec}, 62.8^{\circ} \mathrm{C}$ for $15 \mathrm{sec}, 72^{\circ} \mathrm{C}$ for $20 \mathrm{sec}$ and $72^{\circ} \mathrm{C}$ for $5 \mathrm{~min}$, performed for 35 cycles. The expression of GAPDH was used as the internal control.

Western blot analysis. Seventy-two hours after transfection with the plasmid or siRNA, the cells were harvested and washed with ice-cold phosphate buffer. Cells were lysed in RIPA buffer (150 mmol/1 NaCl, $50 \mathrm{mmol} / \mathrm{l}$ Tris-HCl, $1 \%$ NP-40, $0.1 \%$ sodium dodecyl sulfate, $0.5 \%$ sodium deoxycholate and $1 \mathrm{mmol} / \mathrm{l}$ phenylmethylsulfonyl fluoride). Protein concentrations were determined using the BCA method (Thermo Scientific-Pierce Biotechnology, Inc.). Proteins were resolved in sodium dodecyl sulfate-polyacrylamide gel electrophoresis gels and transferred onto polyvinylidene fluoride membranes (Millipore, Bedford, MA). Membranes were incubated with the following primary antibodies overnight at $4^{\circ} \mathrm{C}$ : Nek2 (1:500; Sigma, St. Louis, MO), $\gamma$-tubulin $(1: 1,000$, Sigma), myc (1:2,000; Cell Signaling Technology, Inc.) and $\beta$-actin (1:2,000; Santa Cruz Biotechnology, Inc.). After incubation with secondary antibodies, immunostained bands were detected by ECL (Thermo Scientific-Pierce Biotechnology, Inc.) western blotting reagents. The expression of $\beta$-actin was used as the internal control.

Cell growth analysis. Cells were cultured in 96-well plates at a density of $1 \times 10^{4}$ cells/well. After transfection, the quantity of viable cells was estimated by a colorimetric assay using 3-(4,5-dimethylthiazol-2-yl)-2,5-diphenyltetrazolium bromide (MTT). MTT (10 $\mu \mathrm{l}$ of $5 \mathrm{mg} / \mathrm{ml}$ solution, Sigma) was added to each well of the titration plate and incubated at $37^{\circ} \mathrm{C}$ for $4 \mathrm{~h}$. The cells were then treated with DMSO (40 $\mu \mathrm{l} /$ well) and incubated at $37^{\circ} \mathrm{C}$ for $1 \mathrm{~h}$. The absorbance of each well was determined at $570 \mathrm{~nm}$. The percentage viability was defined as the relative absorbance of the transfected vs. blank control cells. All experiments were performed with 5-wells/experiment and repeated at least 3 times.

Assessment of percentage of apoptotic cells. To detect apoptotic cells, the cells were stained with DNA binding dye Hoechst 33342 (Sigma). Cells with the indicated treatment 
were fixed with $4 \%$ formaldehyde in phosphate-buffered saline (PBS) for $10 \mathrm{~min}$ at $4^{\circ} \mathrm{C}$ and then washed with PBS. Cells were incubated for $20 \mathrm{~min}$ with $5 \mu \mathrm{g} / \mathrm{ml}$ of Hoechst 33342 (Sigma) to stain the nuclei. After washing with PBS, the apoptotic cells were observed under a confocal fluorescence microscope (Leica TCS SMD FCS, Leica, Wetzlar, Germany) (x100). Cells exhibiting condensed chromatin and fragmented nuclei (Hoechst 33342 stain, blue) were scored as apoptotic cells. For each Hoechst experiment, at least 200 cells in 5 random scope fields were counted for apoptotic rate.

In vitro cell invasion and migration assay. Invasion assay with a Matrigel-coated membrane and migration assay with a Matrigel-uncoated membrane were performed using a 24-well chamber system (BD Biosciences, Bedford, MA), according to the manufacturer's instructions. The cells were trypsinized and seeded in the upper chamber at $2.5 \times 10^{5}$ cells/well in serum-free medium. Medium supplemented with $50 \%$ FBS was placed at the bottom of the wells. Incubation was carried out for $24 \mathrm{~h}$ at $37^{\circ} \mathrm{C}$ in humidified air with a $5 \% \mathrm{CO}_{2}$ atmosphere. The cells were allowed to migrate through a porous, Matrigel-coated or uncoated membrane (BD Biosciences). Following incubation, the chambers were removed, and invading cells on the bottom side of the membrane were fixed with methanol and stained with Giemsa. The number of invading cells or migrating cells were determined by counting 5 high-power fields (x400) on each membrane and calculated as the mean number of cells/ field.

Immunofluorescence microscopy. Cells grown on glass coverslips were fixed with cold methanol for $5 \mathrm{~min}$. Cells were then blocked by $2 \%$ bovine serum albumin (BSA) in PBS for $30 \mathrm{~min}$. Cells were then fixed and double-stained with anti-myc (1:2,000; Cell Signaling Technology, Inc.) or anti$\gamma$-tubulin $(1: 1,000 ;$ Sigma) or anti-centrin 2 antibodies $(1: 50$; Santa Cruz Biotechnology, Inc.). The cells were then incubated with Alexa Fluor 594 goat anti-mouse or Alexa Fluor 488 goat anti-rabbit ( $1 \mathrm{~g} / \mathrm{ml}$; Invitrogen) as the secondary antibodies, followed by staining with 4',6-diamidino-2-phenylindole (DAPI; Biosource) for $5 \mathrm{~min}$. The fluorescence staining intensity was then examined by immunofluorescence microscopy.

Tissue specimens. All cases of breast surgical specimens, including $10 \mathrm{NBT}, 10 \mathrm{ADH}, 10 \mathrm{DCIS}$ and 10 IDC, were anonymized after collection from the Breast Pathology Department, Tianjin Tumor Hospital. Tissues of 10 NBT cases were obtained from the quadrant far away from the original foci in the surgical specimens of 10 cases of IDC. The pathological diagnosis was confirmed by 2 senior pathologists according to the 2003 WHO histological classification of tumors of the breast. Tissue collection and analysis in this study were approved by the Ethics Committee of the Tianjin Medical University Cancer Institute and Hospital, Tianjin, China. Informed consent was obtained from all patients prior to surgery and examination of the specimens.

Statistical analysis. All in vitro experiments were repeated 3 times. Differences were statistically evaluated using the Student's t-test. A P-value $<0.05$ was considered to indicate a statistically significant difference.
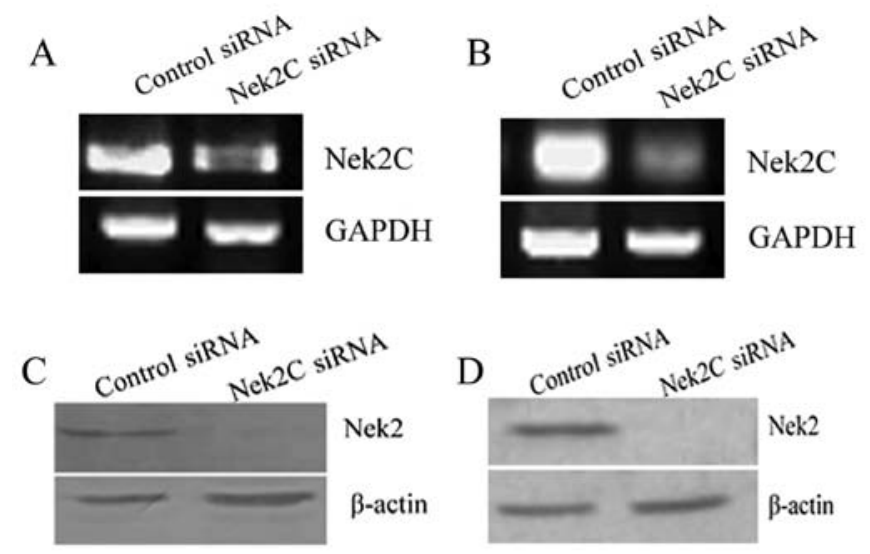

Figure 1. Reduced expression of Nek2C in Nek2C siRNA-transfected breast cancer cells. As shown by RT-PCR analysis, the expression levels of Nek2C mRNA were lower in Nek2C siRNA-transfected (A) MCF10DCIS.com and (B) MCF10CA1a cell lines than in the control siRNA-transfected cells. The expression levels of the Nek2 protein were evaluated by western blot analysis. Nek2 was significantly decreased in (C) MCF10DCIS.com and (D) MCF10CA1a cell lines which were transfected with Nek2C siRNA.

\section{Results}

Effect of Nek2C expression on breast tumor cells. Breast cancer cells (MCF10DCIS.com and MCF10CA1a) were transfected with Nek2C siRNA and a scrambled siRNA, as the negative control (siControl), to study the function of Nek2C. The transcriptional levels of Nek2C were examined by RT-PCR. The reduced expression of Nek2C was observed in the MCF10DCIS.com cells, which were transfected with Nek2C siRNA (siRNA-2), compared to the cells transfected with control siRNA (Fig. 1A). The reduction rates in Nek2C signals were almost similar to those in MCF10CA1a cells (Fig. 1B). Hence, siRNA inhibits Nek2C expression. Western blot analysis was carried out to detect the protein expression of Nek 2 in these breast carcinoma cell lines. The protein levels of Nek2 in the breast cancer cell lines (MCF10DCIS.com and MCF10CA1a), which were transfected with Nek2C siRNA (siRNA-2), were reduced compared to those in the control siRNA-transfected cells (Fig. 1C and D).

We then examined the effect of siRNA treatment on the growth of breast carcinoma cells to determine the effect of the Nek2C knockdown. We found that Nek2C knockdown by RNAi remarkably affected the viability of the MCF10DCIS. com and MCF10CA1a cell lines. The downregulation of Nek2C using Nek2C siRNA blocked cell growth. Marked growth inhibition was evident at $96 \mathrm{~h}$ post siRNA transfection in the MCF10DCIS.com cells (Fig. 2A). The growth of MCF10CA1a cells was also suppressed by Nek2C siRNA treatment to levels similar to those of MCF10DCIS.com cells (Fig. 2B). By contrast, transfection with control siRNA did not alter cell growth significantly. Increased levels of apoptosis were observed in MCF10DCIS.com and MCF10CA1a cell lines after 3-5 days in the absence of Nek2C (Fig. 2C and D). Furthermore, typical apoptotic changes, such as nuclear fragmentation in the Nek2C siRNA-transfected cells were noted, and the number of apoptotic cells was significantly greater compared to the cells transfected with the control siRNA. Thus, apoptosis is possibly the mechanism by which cell numbers are reduced. 



Figure 2. Effect of Nek2C siRNA on the growth and apoptosis of breast cancer cells. (A) The number of MCF10DCIS.com cells, which were transfected with Nek2C siRNA, was reduced compared to the control siRNA-transfected cells. (B) In the MCF10CA1a cell, the growth reduction rates were almost similar to those in the MCF10DCIS.com cells. Thus, the number of apoptotic cells in the Nek2C siRNA-transfected (C) MCF10DCIS.com and (D) MCF10CA1a cells was significantly increased compared to that of the control cells $(\mathrm{P}<0.05)$. The values in the figures are expressed as the means \pm standard deviation $(\mathrm{SD})$. Experiments were repeated at least 3 times to insure the consistency of the results.

Moreover, after 24-h invasion, the invasion of breast cancer cells was significantly attenuated in the MCF10DCIS.com and MCF10CA1a cell lines which were transfected with Nek2C siRNA compared to the control (Fig. 3A and B) $(\mathrm{P}<0.05)$. Furthermore, the forced downregulation of Nek2C in these cell lines significantly suppressed their migration through the Transwell membrane (Fig. 3C and D) $(\mathrm{P}<0.05)$. Collectively, these results imply a pivotal role of Nek2C in tumor progression and aggressiveness. The knockdown of Nek2C significantly suppresses tumor cell invasion and migration. Thus, the reduction in Nek2C expression attenuates the progression to an invasive tumor and impairs the growth of tumors that ultimately form.

Role of the Nek2C plasmid in breast cells. The pcDNAmycNek $2 \mathrm{C}$ and pcDNA3.0-myc vectors were transfected into the breast cell lines, MCF10A (Fig. 4C) and MCF10AT (Fig. 4D). First, we examined the expression levels of Nek2C in MCF10A and MCF10AT cells by semiquantitative RT-PCR. The mRNA expression levels of Nek2C were significantly upregulated in Nek2C-transfected MCF10A and MCF10AT cells compared to those of the control cells (Fig. 5A and B). The protein expression levels of Nek2C were then detected by western blot analysis in these cells. Similarily, the expression levels of Nek2C proteins were markedly higher in the Nek2C- transfected MCF10A and MCF10AT cells than in the control cells (Fig. 5C and D). We focused on the role of Nek2C in subsequent experiments.

In parallel, cell viability was measured for 7 days following transfection. pcDNA3.1-Nek2C had a detectable effect on the viability of the breast cancer cell lines. The growth of MCF10A cells was substantially promoted by the Nek2C plasmid transfection compared with that of the cells transfected with the control empty vector (Fig. 6A). The growth of MCF10AT cells was also increased by the Nek2C plasmid to levels similar to those of MCF10A cells (Fig. 6B). These findings suggest that Nek2C plays an important role in the growth and survival of MCF10A and MCF10AT cells.

A peculiar feature of the expression of Nek2C in human breast cells was its predominantly nuclear localization. To further investigate the localization of Nek2 $\mathrm{C}$ in human breast cell lines, we used the MCF10AT cell line, which was transfected with the Nek2C plasmid. First, western blot analysis confirmed that the Nek2C protein was upregulated in Nek2Ctransfected MCF10AT cells with respect to the non-transformed Nek2C cells. Moreover, subcellular localizations of the proteins were determined by immunocytochemistry with the specific antibodies. Immunofluorescence analysis confirmed that the Nek2C protein was predominantly present in the nucleus in Nek2C-transfected MCF10AT cells (Fig. 7). 
A

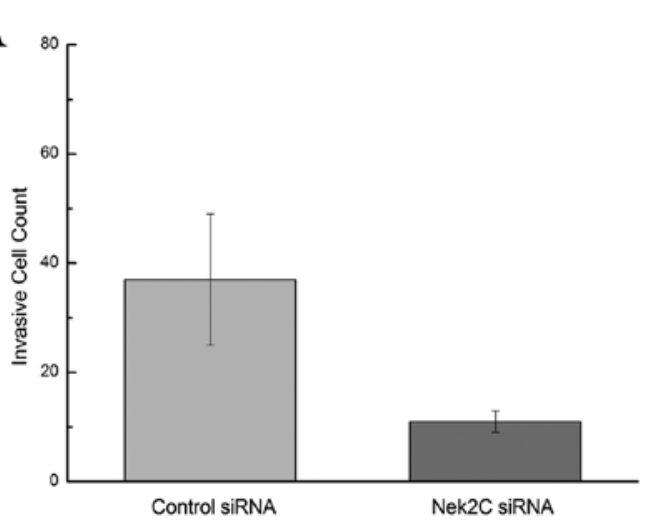

$\mathrm{C}$



B

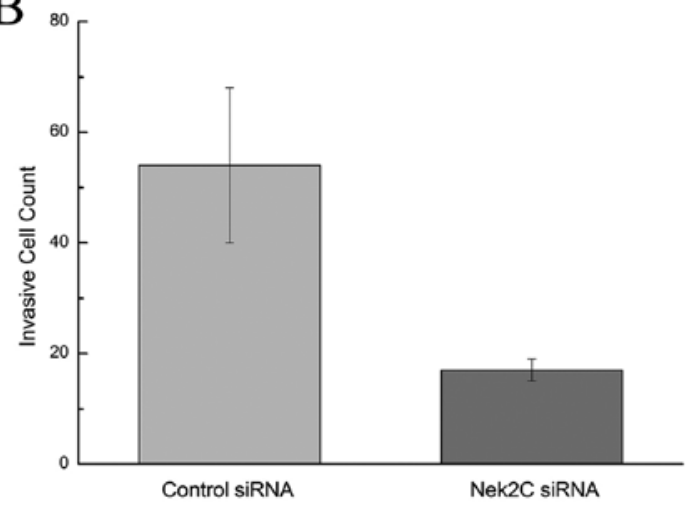

$\mathrm{D}$



Figure 3. Nek2C promoted human breast cancer cell invasion and migration. Invasion analysis of MCF10DCIS.com and MCF10CA1a cells treated with Nek2C siRNA. Nek2C siRNA significantly suppressed tumor cell invasion in (A) MCF10DCIS.com and (B) MCF10CA1a cell lines (P<0.05). Migration analysis of MCF10DCIS.com and MCF10CA1a cells treated with Nek2C siRNA. Compared with the negative control cells, the migrated number of (C) MCF10DCIS.com and (D) MCF10CA1a cells was significantly reduced by treatment with Nek2C siRNA $(\mathrm{P}<0.05)$. The values in the figures are expressed as the means \pm SD. Experiments were repeated at least 3 times to insure the consistency of the results.

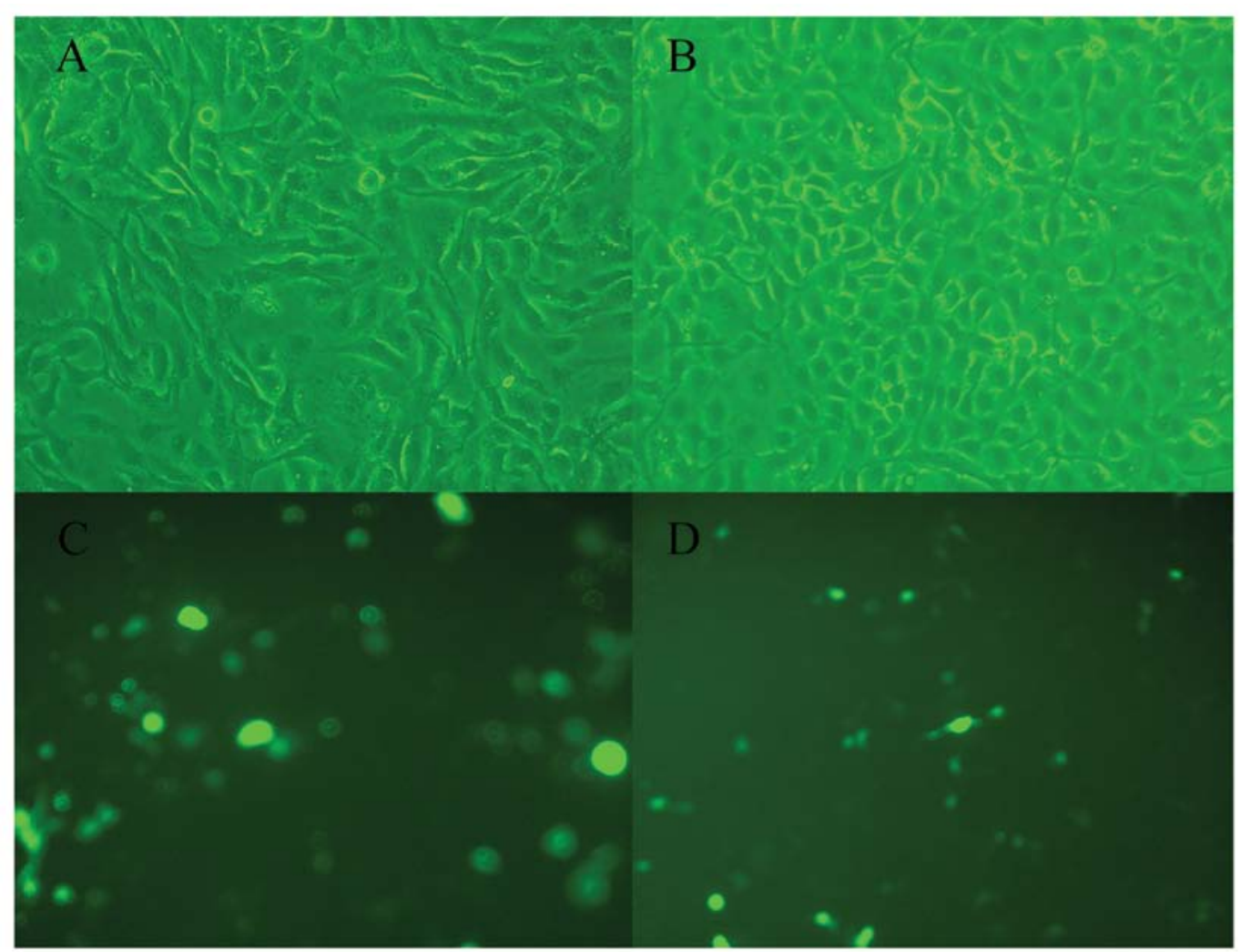

Figure 4. Phase contrast images of (A and C) MCF10A and (C and D) MCF10AT cells (magnification, x200). Prior to pcDNA-mycNek2C transfection, (A) MCF10A and (B) MCF10AT cell lines grew continuously. After transfection, (C) MCF10A and (D) MCF10AT cells were observed using a fluorescence microscope. 
A
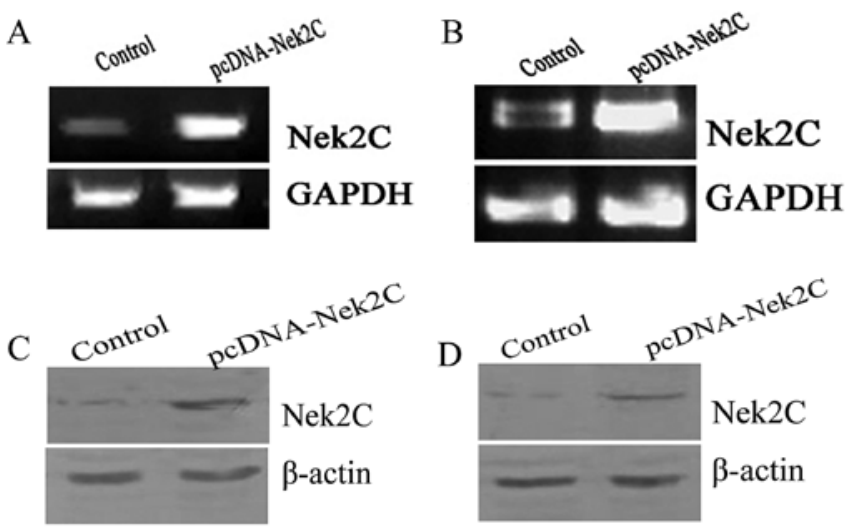

Figure 5. Expression of Nek2C candidate targets at $72 \mathrm{~h}$ after the transfection of MCF10A and MCF10AT cells with the Nek2C plasmid, as assessed by RT-PCR and western blot analysis. $\beta$-actin or GAPDH were used as the internal control. The mRNA and protein expression levels of Nek2C were significantly upregulated in Nek2C-transfected (A and C) MCF10A and (B and D) MCF10AT cells compared with the control cells. All experiments were repeated and yielded similar results.

Expression of Nek2C in breast tissue. The MCF10DCIS.com and MCF10CA1a cell lines displayed similar expression levels of Nek2C-mRNA, with Nek2C consistently expressed at high levels. We analyzed 40 breast samples, including 10 NBT, $10 \mathrm{ADH}, 10 \mathrm{DCIS}$ and $10 \mathrm{IDC}$ samples in order to determine whether the mRNA expression levels of the Nek2C kinase were altered in human breast tissue. Both tumor and normal tissues were examined to compare the mRNA expression levels using RT-PCR. We found that Nek2C-mRNA was more abundant in extracts obtained from IDC and DCIS samples than in those obtained from NBT or samples from a patient affected by ADH. Nine samples (90\%) exhibited a significantly elevated Nek2C mRNA expression in IDC, whereas $1(10 \%)$ showed no significant difference with the normal tissue. In the DCIS cases, 9 samples (90\%) showed an elevated Nek2C mRNA expression levels compared with NBT. Thus, human Nek2C is expressed at high levels in DCIS and IDC, in contrast to its low levels in NBT (Fig. 8A). These results indicate that Nek2C mRNA is frequently upregulated in human breast cancer. We also used RT-PCR to evaluate the quantity of Nek2C in correlation with tumor grade. The expression of Nek2C in high-grade (grade III) tumor tissue (Fig. 8B) was significantly higher than in low-grade (grade I and II) tumor tissue. Hence, Nek2C affects tumorigenesis and progression in breast cancer.

\section{Discussion}

Breast carcinoma is one of the leading causes of morbidity in women. The prognosis for this disease is generally very poor, with a high mortality rate. Nek2 in humans is expressed as at least 3 splice variants, namely, Nek2A, Nek2B and Nek2C. Nek2A is one of the main splice variants of Nek2, and is considered of great importance in revealing the molecular mechanisms of carcinogenesis $(15,16)$. Nek2C (originally known as Nek2A-T) was identified after yeast two-hybrid screening of a testis library, with PP $1 \gamma 1$ and PP $1 \gamma 2$ as bait (12). Nek2C differs from Nek2A, as the former lacks 8 amino acids (371-378) in the middle of the non-catalytic domain. However,


Figure 6. Cell viability was determined by MTT assay. The growth of the (A) MCF10A and (B) MCF10AT cells, which were transfected by the Nek2C plasmid, was boosted by the overexpression of Nek2C compared to the control plasmid-transfected cells. Results are the means \pm SD of 3 independent experiments.

as our results suggest, this small difference has significant consequences on subcellular localization, which in turn may allow the different splice variants to undertake distinct functions during cell division.

In breast carcinoma, Her-2/neu (17), Myc (18) and Wnt (19) are potential candidate genes for oncogene addiction. In this study, we demonstrate that the Nek2C pathway is necessary to maintain the tumorigenic growth of breast carcinoma cells, suggesting Nek2C to be another candidate gene for oncogene addiction. The effectiveness of Nek2C as a chemotherapeutic target is worth considering. Nek2C expression is frequently elevated in cancer cells, possibly due to gene amplification or loss of transcriptional control. Moreover, immunofluorescence analysis has confirmed that $\mathrm{Nek} 2 \mathrm{C}$ is located in the nuclear region (15). Hence, an additional role of Nek2C in the regulation of nuclear events in breast cancer cells is suggested. The resulting protein contains a novel nuclear localization sequence that is absent in Nek2A and Nek2B. Although experiments with recombinant proteins have shown that Nek2A also partially accumulates in the nuclei, the non-centrosomal pool of Nek2C accumulates in the nuclei more efficiently. Given the similar size and the lack of specific antibodies, endogenous Nek2A and Nek2C cannot be distinguished at the protein level. The preferential uptake of Nek2C into the nucleus is evident, 

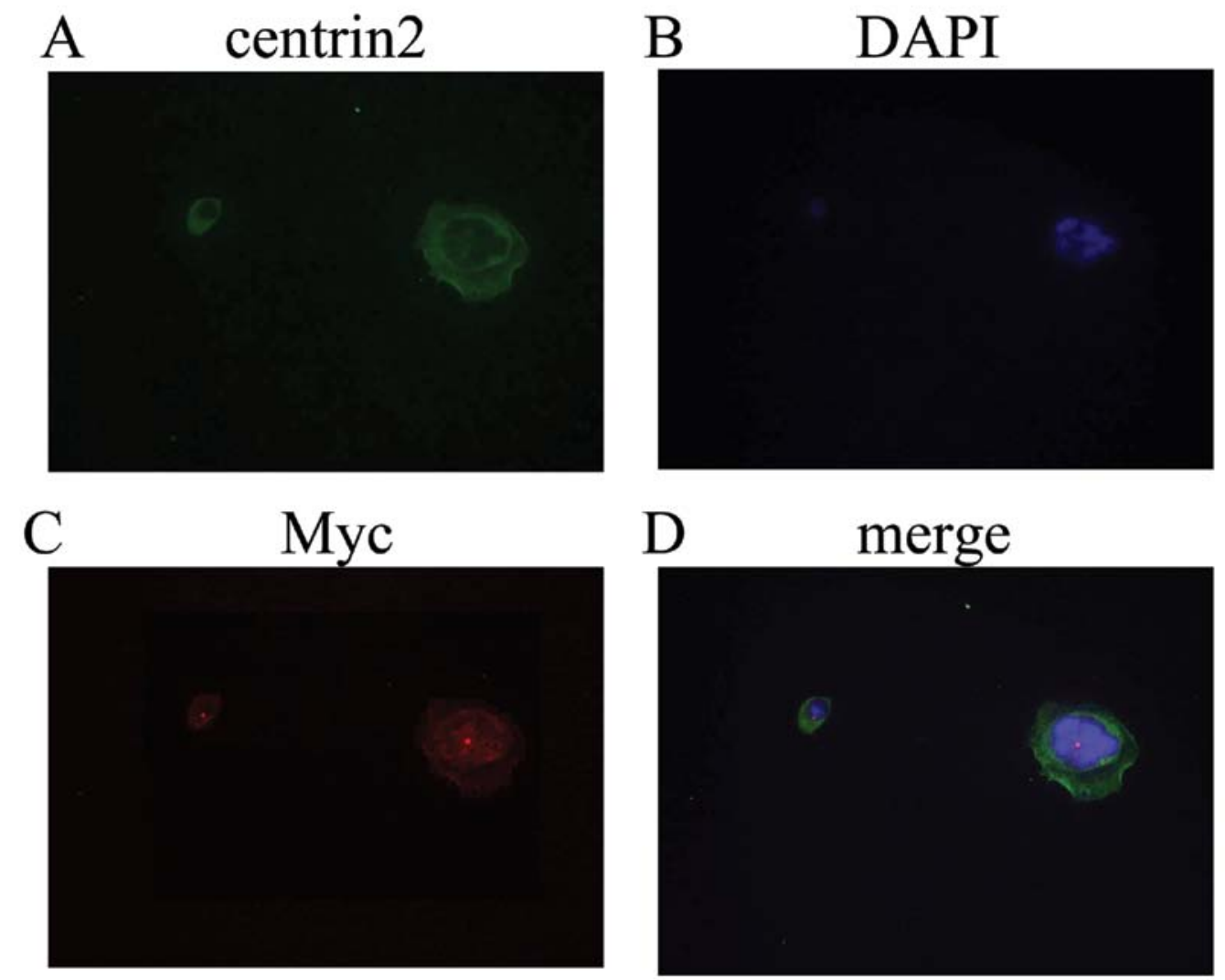

Figure 7. Immunofluorescence of Nek2C in the Nek2C plasmid-transfected MCF10AT cells (magnification, $\mathrm{x} 200$ ). Immunofluorescence microscopy of MCF10AT cells transfected with myc-tagged Nek2C and stained with antibodies against the myc-tag (red) and centrin2 (green). DNA was stained with DAPI (blue). The results showed that the Nek2C protein was predominantly present in the nucleus in Nek2C-transfected MCF10AT cells.

A

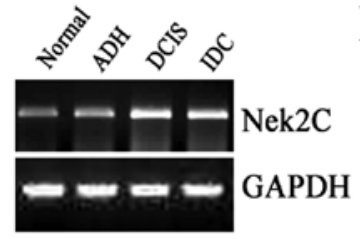

B

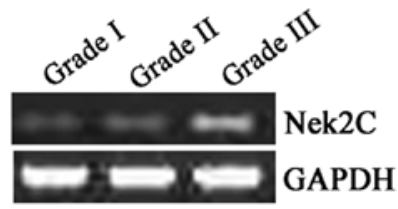

Figure 8. The expression of $\mathrm{Nek} 2 \mathrm{C}$ in breast tissue. (A) Examination of mRNA levels by RT-PCR also indicated that Nek2C expression was significantly elevated in IDC and DCIS tissues relative to the normal and ADH tissues at the transcriptional level. (B) The expression of Nek2C in high-grade tumor tissue was higher than in low-grade tumor tissue.

whereas Nek2A is more evenly distributed throughout the cell. This phenomenon can be explained by the loss of the 8 amino acids from Nek2C resulting from splicing. Thus, a specific antibody that can distinguish between Nek2A and Nek2C is required to further investigate the localization of Nek2C.

RNA interference has emerged as a natural and highly efficient mechanism for gene silencing (20-22). A number of previous studies have reported on the consequences of Nek2 depletion using RNA interference strategies $(1,23-25)$. In this study, we treated MCF10DCIS.com and MCF10CA1a cells with double-stranded siRNA oligonucleotides against Nek2C to study the function of Nek2C. The expression of Nek2C in MCF10DCIS.com and MCF10CA1a cells was substantially suppressed by Nek2C siRNA treatment, but not by control
siRNA. We also found that the treatment of MCF10DCIS.com and MCF10CA1a cells with Nek2C siRNA caused concomitant suppression of cell growth, invasion and migration compared to the control siRNA-treated cells. These findings bear some similarities to the results of Tsunoda et al (26) and Westwood et al (27). The results showed that breast carcinoma cells have a specific dependence on Nek2C for their tumorigenic growth. Patients with breast cancer generally receive chemotherapy treatment with paclitaxel, platinum-based agents, or a combination of both. Hence, developing a novel strategy for breast cancer treatment is necessary and urgent. To the best of our knowledge, this study has demonstrated for the first time that $\mathrm{Nek} 2 \mathrm{C}$ plays a pivotal role in tumor progression, and that $\mathrm{Nek} 2 \mathrm{C}$ inhibition can significantly suppress tumorigenesis and progression in breast cancer, which may imply a novel therapeutic target. Nek2C depletion suppresses not only the anchorage-independent growth but also the invasiveness of breast carcinoma. Although the effect of Nek2C siRNA in breast carcinoma is quite promising, whether the effect of siRNA is limited to breast carcinoma remains unclear.

We used MCF10A and MCF10AT breast cancer cell lines that maintain the features of the original breast cells. Myc-tagged Nek2C was transfected into these 2 cell lines. RT-PCR analysis confirmed that Nek2C mRNA was upregulated in the transfected MCF10A and MCF10AT cells compared to the control cells. The 2 types of breast cells transfected with the Nek2C plasmid showed very similar expression levels of Nek2C proteins, with Nek2C consistently expressed at higher 
levels than in the non-transfected cells. We examined the effect of exogenous Nek2C expression in 'normal' immortalized breast cells (MCF10A) and (MCF10AT) on the growth of breast cancer cells and found that the expression of Nek2C stimulated the growth of these cells.

We therefore analyzed Nek2C mRNA expression in samples of human breast tissue using RT-PCR. Strikingly, Nek2C mRNA expression was elevated significantly in most of these breast tumor samples. Elevated Nek2C expression was clearly detected in DCIS and IDC tissues compared with those in NBT or ADH. Moreover, analysis of IDC tissue also revealed a significant increase in the expression of Nek2C mRNA with tumor grade increase. Thus, Nek2C may be a contributory factor in cancer malignant progression.

In this study, we show that Nek2C is abundantly expressed in breast cancer cells. This protein is required for the tumorigenic growth and survival of these cells. To the best of our knowledge, we show for the first time that siRNA against Nek2C substantially suppresses the growth, in vitro invasiveness and migration of breast carcinoma cell lines. Although the precise mechanism by which Nek2C siRNA suppresses tumor growth remains unclear, our results strongly suggest the necessity of Nek2C in the tumorigenesis of breast carcinoma cells. To the best of our knowledge, this is the first report demonstrating that $\mathrm{Nek} 2 \mathrm{C}$ plays a critical role in carcinogenesis, tumor invasion and the tumorigenic growth of breast carcinoma. Nek2C mRNA was overexpressed in both breast cancer cell lines and patient tissue samples. The contribution of this upregulation in tumor progression should be elucidated as the elevated expression of Nek2C may drive tumorigenesis, and a correlation exists between increased Nek2C and poor patient outcome. Therefore, further research is required to determine the role of $\mathrm{Nek} 2 \mathrm{C}$ in human breast cancer.

\section{Acknowledgements}

This study was financially supported by the National Science Foundation of China (30872519); the Scientific and Technological Development Fund of the Tianjin Scientific and Technological Committee (09JCYBJC10100); the Program for Chang-jiang Scholars and Innovative Research Team in University (IRT0743).

\section{References}

1. Fletcher L, Cerniglia GJ, Nigg EA, Yend TJ and Muschel RJ: Inhibition of centrosome separation after DNA damage: a role for Nek2. Radiat Res 162: 128-135, 2004.

2. Fry AM: The Nek2 protein kinase: a novel regulator of centrosome structure. Oncogene 21: 6184-6194, 2002.

3. O'Connell MJ, Krien MJ and Hunter T: Never say never. The NIMA-related protein kinases in mitotic control. Trends Cell Biol 13: 221-228, 2003.

4. Fry AM, Meraldi P and Nigg EA: A centrosomal function for the human Nek2 protein kinase, a member of the NIMA family of cell cycle regulators. EMBO J 17: 470-481, 1998.

5. Guo HQ, Gao M, Ma J, Xiao T, Zhao LL, Gao Y and Pan QJ: Analysis of the cellular centrosome in fine-needle aspirations of the breast. Breast Cancer Res 9: R48, 2007.
6. Lingle WL, Lutz WH, Ingle JN, Maihle NJ and Salisbury JL: Centrosome hypertrophy in human breast tumors: Implications for genomic stability and cell polarity. Proc Natl Acad Sci USA 95: 2950-2955, 1998

7. Liu Q, Hirohashi Y, Du X, Greene MI and Wang Q: Nek2 targets the mitotic checkpoint proteins Mad2 and Cdc20: a mechanism for aneuploidy in cancer. Exp Mol Pathol 88: 225-233, 2010.

8. Hayward DG and Fry AM: Nek2 kinase in chromosome instability and cancer. Cancer Lett 237: 155-166, 2006.

9. Hayward DG, Clarke RB, Faragher AJ, Pillai MR, Hagan IM and Fry AM: The centrosomal kinase Nek2 displays elevated levels of protein expression in human breast cancer. Cancer Res 64: 7370-7376, 2004.

10. de Vos S, Hofmann WK, Grogan TM, et al: Gene expression profile of serial samples of transformed B-cell lymphomas. Lab Invest 83: 271-285, 2003.

11. Uto K, Nakajo N and Sagata N: Two structural variants of Nek2 kinase, termed Nek2A and Nek2B, are differentially expressed in Xenopus tissues and development. Dev Biol 208: 456-464, 1999.

12. Fardilha $\mathrm{M}, \mathrm{Wu} \mathrm{W}, \mathrm{Sa} \mathrm{R}$, et al: Alternatively spliced protein variants as potential therapeutic targets for male infertility and contraception. Ann NY Acad Sci 1030: 468-478, 2004.

13. Worsham MJ, Pals G, Schouten JP, Miller F, Tiwari N, van Spaendonk R and Wolman SR: High-resolution mapping of molecular events associated with immortalization, transformation, and progression to breast cancer in the MCF10 model. Breast Cancer Res Treat 96: 177-186, 2006.

14. Vazquez-Martin A, Colomer R, Brunet J, Lupu R and Menendez JA: Overexpression of fatty acid synthase gene activates HER1/HER2 tyrosine kinase receptors in human breast epithelial cells. Cell Prolif 41: 59-85, 2008.

15. Wu W, Baxter JE, Wattam SL, et al: Alternative splicing controls nuclear translocation of the cell cycle-regulated Nek 2 kinase. J Biol Chem 282: 26431-26440, 2007.

16. Wang S, Li W, Liu N, et al: Nek2A contributes to tumorigenic growth and possibly functions as potential therapeutic target for human breast cancer. J Cell Biochem 113: 1904-1914, 2012.

17. Moody SE, Perez D, Pan TC, et al: The transcriptional repressor Snail promotes mammary tumor recurrence. Cancer Cell 8: 197-209, 2005.

18. D'Cruz CM, Gunther EJ, Boxer RB, et al: c-MYC induces mammary tumorigenesis by means of a preferred pathway involving spontaneous Kras2 mutations. Nat Med 7: 235-239, 2001.

19. Gunther EJ, Moody SE, Belka GK, et al: Impact of p53 loss on reversal and recurrence of conditional Wnt-induced tumorigenesis. Genes Dev 17: 488-501, 2003.

20. Karagiannis TC and El-Osta A: RNA interference and potential therapeutic applications of short interfering RNAs. Cancer Gene Ther 12: 787-795, 2005.

21. Lu PY, Xie F and Woodle MC: In vivo application of RNA interference: from functional genomics to therapeutics. Adv Gen 54: 117-142, 2005.

22. Ameyar-Zazoua M, Guasconi V and Ait-Si-Ali S: siRNA as a route to new cancer therapies. Expert Opin Biol Ther 5: 221-224, 2005.

23. Fletcher L, Cerniglia GJ, Yen TJ and Muschel RJ: Live cell imaging reveals distinct roles in cell cycle regulation for Nek2A and Nek2B. Biochem Biophys Acta 1744: 89-92, 2005.

24. Lou Y, Yao J, Zereshki A, et al: NEK2A interacts with MAD1 and possibly functions as a novel integrator of the spindle checkpoint signaling. J Biol Chem 279: 20049-20057, 2004.

25. Yao J, Fu C, Ding X, et al: Nek2A kinase regulates the localization of numatrin to centrosome in mitosis. FEBS Lett 575: 112-118, 2004.

26. Tsunoda N, Kokuryo T, Oda K, et al: Nek2 as a novel molecular target for the treatment of breast carcinoma. Cancer Sci 100: 111-116, 2009.

27. Westwood I, Cheary DM, Baxter JE, Richards MW, van Montfort RL, Fry AM and Bayliss R: Insights into the conformational variability and regulation of human Nek2 kinase. J Mol Biol 386: 476-485, 2009. 